\title{
Effect of aspect ratio on natural convection in attics subject to periodic thermal forcing
}

\author{
$\begin{array}{lll}\text { S. C. Saha } & \text { C. } \text { Lei }^{2} & \text { J. C. Patterson } \\ \end{array}$
}

(Received 30 August 2006; revised 9 December 2007)

\begin{abstract}
We consider the heat transfer through the attics of buildings under realistic (periodic) thermal forcing. The objective of this study is to investigate numerically the effect of the aspect ratio (height to base ratio) on the heat transfer through the attics. A fixed Grashof number $1.33 \times 10^{6}$ is considered for three different aspect ratios $0.2,0.5$ and 1.0. The Prandtl number is also fixed at 0.71 for air. The details of the numerical model as well as the flow structures and heat transfer data are presented.
\end{abstract}

\section{Contents}

1 Introduction

C678

See http://anziamj.austms.org.au/ojs/index.php/ANZIAMJ/article/view/109 for this article, (c) Austral. Mathematical Soc. 2007. Published December 20, 2007. ISSN 1446-8735 
3 Results and Discussion

C682

3.1 The overall flow response . . . . . . . . . . . . . C682

3.2 Effects of aspect ratio on the flow response . . . . . . . C685

3.3 Heat transfer . . . . . . . . . . . . . . . . C687

4 Conclusion

C688

References

C690

\section{Introduction}

The attic space between the roof and the ceiling of a building is responsible for a significant portion of the heat transfer to and from the building. The heat transfer through attics is mainly governed by a natural convection process, and affected by factors including the geometry, the interior structure and the insulation. A few publications are devoted to laminar natural convection in two dimensional isosceles triangular cavities in the vast literature on convection heat transfer.

The temperature and flow patterns, local wall heat fluxes and mean heat flux were measured experimentally by Flack $[1,2]$. The triangular cavities, filled with air, were heated/cooled from the base and cooled/heated from the sloping walls covering a wide range of Grashof numbers. Flack $[1,2]$ also reported the critical Grashof numbers of the transition from laminar to turbulent regimes of the flow. Akinsete and Coleman [3] numerically simulated the attic space with a hot upper sloping wall and a cooled base. Their aim was to obtain previously unavailable heat transfer data relevant to air conditioning calculations.

Poulikakos and Bejan [4] reported an analytical investigation in the form 
of a scale analysis and a numerical study of the fluid mechanics inside a right triangular cavity with a cold upper inclined wall, a warm horizontal bottom wall and an insulated vertical wall. Del Campo et al. [5] examined the entire isosceles triangular cavities for seven possible combinations of hot wall, cold wall and insulated wall using the finite element method based on a stream function and vorticity formulation.

Holtzmann et al. [7] modelled the buoyant airflow in isosceles triangular cavities with a heated bottom base and symmetrically cooled top sides. They also conducted flow visualization studies with smoke injected into the cavity. The main objective of their experiment was to validate of their numerical prediction of a symmetry breaking bifurcation of the heated air currents that arise with gradual increments in Grashof number.

From the above review we have found that the previous researchers considered either day-time or night-time boundary conditions. However, real buildings are subject to alternative heating and cooling over diurnal cycles. Hence, to model a real attic, periodic thermal forcing is imposed on the sloping boundary in the present study. The effect of the aspect ratio on the flow response and heat transfer is investigated. We describe the formulation of the problem and details of numerical scheme in Section 2 and the results with discussion in Section 3.

\section{Problem formulation}

The physical system is sketched in Figure 1, which is an air filled isosceles triangular cavity of variable aspect ratio. Here $2 L$ is the length of the base or ceiling, $T_{0}$ is the temperature applied on the base, $T_{A}$ is the amplitude of temperature fluctuation on the inclined surfaces, $H$ is the height of the enclosure, and $P$ is the period of the thermal forcing.

Under the Boussinesq approximations the governing continuity, momen- 


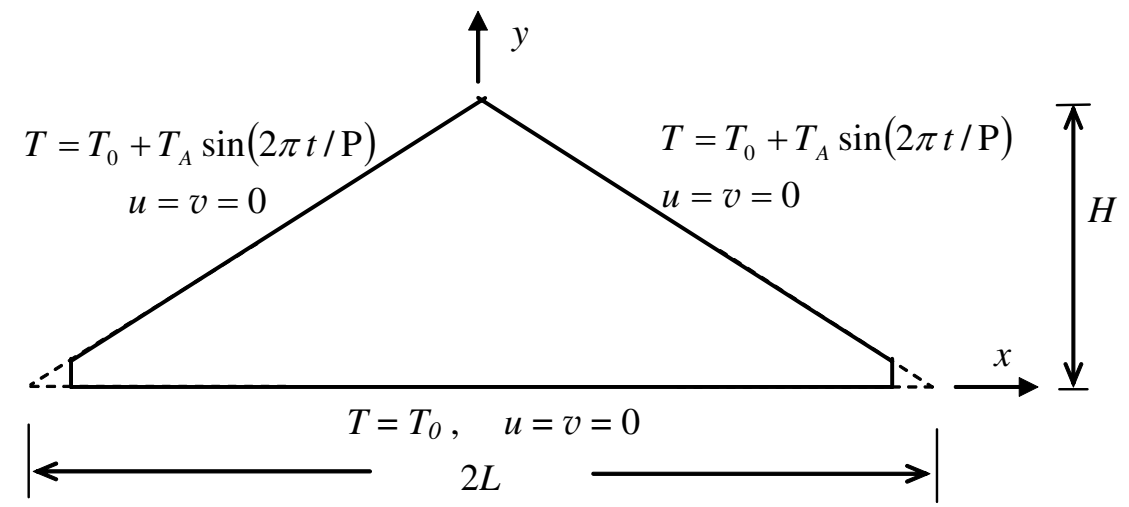

FiguRE 1: A schematic of the geometry and boundary conditions of the enclosure.

tum and energy equations take the following forms:

$$
\begin{aligned}
& \frac{\partial u}{\partial x}+\frac{\partial v}{\partial y}=0 \\
& \frac{\partial u}{\partial t}+u \frac{\partial u}{\partial x}+v \frac{\partial u}{\partial y}=-\frac{1}{\rho} \frac{\partial p}{\partial x}+\nu \nabla^{2} u \\
& \frac{\partial v}{\partial t}+u \frac{\partial v}{\partial x}+v \frac{\partial v}{\partial y}=-\frac{1}{\rho} \frac{\partial p}{\partial y}+\nu \nabla^{2} v+g \beta\left(T-T_{0}\right) \\
& \frac{\partial T}{\partial t}+u \frac{\partial T}{\partial x}+v \frac{\partial T}{\partial y}=\kappa\left(\frac{\partial^{2} T}{\partial x^{2}}+\frac{\partial^{2} T}{\partial y^{2}}\right)
\end{aligned}
$$

where $u$ and $v$ are the velocity components along $x$ - and $y$-directions, $t$ is the time, $p$ is the pressure, $\nu, \rho, \beta$ and $\kappa$ are kinematic viscosity, density of the fluid, coefficient of thermal expansion and thermal diffusivity respectively, $g$ is the acceleration due to gravity and $T$ is the temperature.

The boundary conditions for the present numerical simulation are also shown in Figure 1. Here, the temperature of the bottom wall of the cavity is fixed at $T=T_{0}=295 \mathrm{~K}$ (Kelvin). A periodic temperature boundary 
condition is applied to the two inclined walls with a period of $2000 \mathrm{~s}$ and an amplitude of $T_{A}=5 \mathrm{~K}$. The period is determined by the scaling predictions of Paulikakos and Bejan [4], which shows that the time for the adjustment of the temperature in the thermal boundary layer is far shorter than the thermal forcing period of 24 hours in field situations. According to that study, the growth time of the thermal boundary layer for the present model is of the order of $1 \mathrm{~s}$, and thus the selection of the period of $2000 \mathrm{~s}$ for the thermal forcing is deemed appropriate. Further scaling analysis relevant to the periodic thermal forcing is currently under way. Initially, the fluid in the cavity is motionless and isothermal with a temperature of $T_{0}=295 \mathrm{~K}$. Three governing parameters are the aspect ratio, Grashof number and Prandtl number:

$$
A=\frac{H}{L}, \quad \mathrm{Gr}=\frac{g \beta \Delta T H^{3}}{\nu^{2}}, \quad \operatorname{Pr}=\frac{\nu}{\kappa} .
$$

Three aspect ratios $0.2,0.5$ and 1.0 with a fixed Grashof number $1.33 \times 10^{6}$ and a fixed Prandtl number 0.71 are considered in the present investigation. Based on Flack's [2] experimental observation, which reported the critical Grashof number for the flow to become turbulent, we chose $\mathrm{Gr}=1.33 \times 10^{6}$ so that the flow stays laminar. In reality the Grashof number is much higher than this and an appropriate turbulence model should be applied which is beyond the scope of this study. In order to avoid the singularities at the tips in the numerical simulation, the tips are cut off by $5 \%$ and at the cutting points (refer to Figure 1) rigid non-slip and adiabatic vertical walls are assumed. We anticipate that this modification of the geometry does not alter the overall flow development significantly.

Equations (2)-(4) are solved along with the initial and boundary conditions using the SIMPLE scheme. Mesh and time step dependence tests have been carried out for each aspect ratio. The time steps have been chosen in such a way that the CFL number remains the same for all meshes. The numerical results obtained using different meshes are compared in terms of temperatures recorded at several locations in the enclosure. The comparisons show a maximum temperature variation of about $4 \%$ for different meshes at 
the time $t=0.75 P$ at which the flow in the enclosure is dominated by rising and sinking plumes, and is most sensitive to the mesh. For brevity the detailed results are not presented here. Based on the mesh and time step dependence tests, meshes of $360 \times 90,720 \times 160$ and $270 \times 90$ are selected for aspect ratios $0.2,0.5$ and 1.0 respectively in the present investigation. The corresponding time steps are $0.5 \mathrm{~s}$ for the aspect ratios of 0.2 and 0.5 , and $0.5 \mathrm{~s}$ for the aspect ratio 1.0 .

\section{Results and Discussion}

\subsection{The overall flow response}

Since the initial flow is assumed to be isothermal and motionless, there is a start-up component of the flow response. In order to minimise the startup effect, three full thermal forcing cycles are calculated in the numerical simulation before considering the flow. We found that the start-up effect for the present case is negligible, and the flow response in the third cycle is identical to that in the second cycle. In the following discussion, the results of the third cycle are presented.

Figure 2 shows the snapshots of streamlines and the corresponding isotherms at different stages of the cycle for aspect ratio 1.0. The flow and temperature structures shown in Figure 2(a) for $t=2.00 P$ represent those at the beginning of the daytime heating process in the third thermal forcing cycle. At this time, the inclined surfaces and the bottom surface of the enclosure have the same temperature, but the temperature inside the enclosure is lower than the temperature on the boundaries due to the cooling effect in the previous thermal cycle. The residual temperature structure, which is formed in the previous cooling phase, is still present here. The streamline contours in Figure 2(a) show two circulating cells, and the temperature contours indicate stratification in the upper and lower sections of the enclosure with two cold 

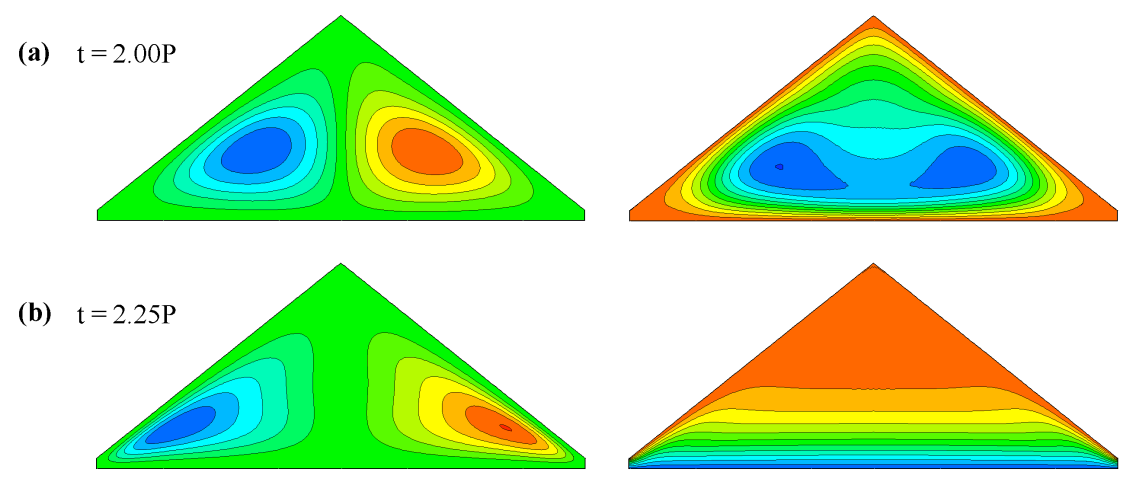

(c) $\mathrm{t}=2.40 \mathrm{P}$
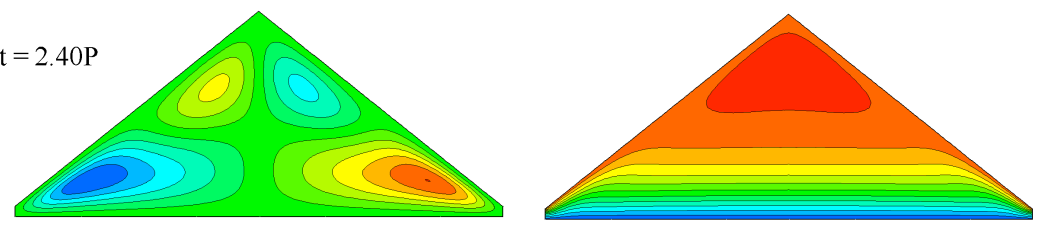

(d) $\mathrm{t}=2.70 \mathrm{P}$
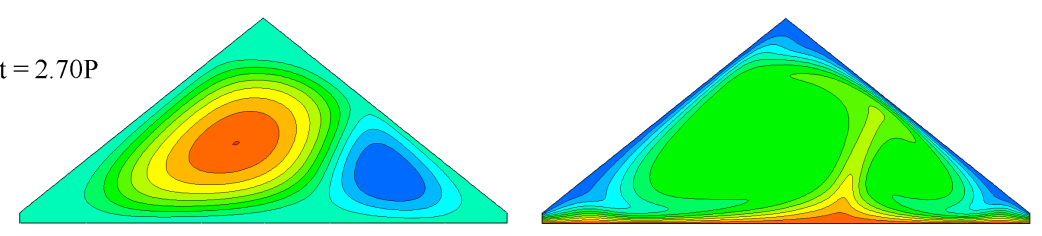

(e) $\mathrm{t}=2.8125 \mathrm{P}$
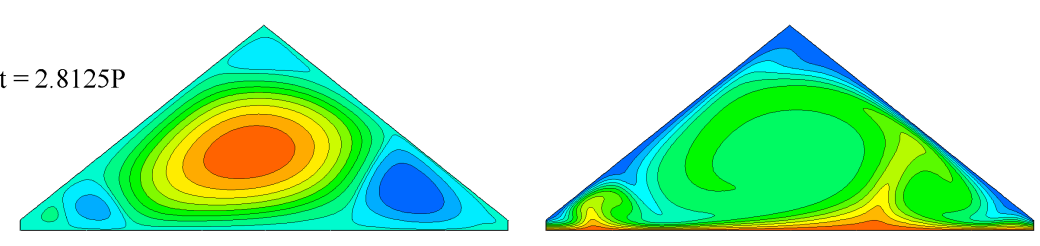

(f) $\mathrm{t}=2.925 \mathrm{P}$

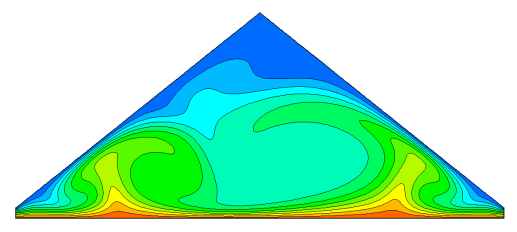

Figure 2: Stream function (left) and isotherms (right) of the third cycle at different times for $A=1.0$ and $\mathrm{Gr}=1.33 \times 10^{6}$. 
regions at the centre.

As the upper surface temperature increases, a distinct temperature stratification is established throughout the enclosure. At $t=2.25 \mathrm{P}$, see Figure 2(b), the temperature on the inclined surfaces peaks. The streamlines at this stage indicate that the centres of the two circulating cells have shifted closer to the tips and the contours are packed near to the inclined surfaces, indicating a strong conduction effect near those boundaries.

From this time the temperature on the inclined surfaces starts to drop, representing a decreasing heating effect. Since the interior flow is stably stratified prior to $t=2.25 \mathrm{P}$, the decrease of the temperature at the inclined surface results in a cooling event, appearing first at the pitchfork and expanding downwards as the surface temperature drops further. At $t=2.40 P$, two additional circulating cells have formed in the upper region of the enclosure, see Figure 2(c), and the newly formed cells push the existing primary cells downwards. The temperature contours in Figure 2(c) show two distinct regions, an expanding upper region responding to the cooling effect, and a shrinking lower region with stratification responding to the decreasing heating effect. By the time $t=2.50 P$ (for brevity the figure is not included), the daytime heating ceases, the lower stratified flow region has disappeared completely and the flow in the enclosure is dominated by the cooling effect. At this time, the top and bottom surfaces again have the same temperature, but the interior temperature is higher than that on the boundaries.

As the upper inclined surface temperature drops below the bottom surface temperature, $t=2.70 P$ shown in Figure 2(d), the cold-air layer under the inclined surfaces becomes unstable. In the meantime, the hot-air layer above the ceiling also becomes unstable. As a consequence, sinking cold-air plumes and rising hot-air plumes are visible in the isotherm contours and a cellular flow pattern is formed, as shown in the stream function contours (see Figure 2d). Notice that the flow is asymmetric about the geometric symmetry plane at this time. The large cell from the left hand side of the centreline is pushing the right cell towards the right tip and becoming larger. 
The central plume is shifted to one side, rises up to meet the cold sloping wall, and continues upwards toward the top of the enclosure. This large velocity moves warm air from the bottom of the geometry upwards and at the same time cold air from the top downwards. The observed asymmetric flow structure in the isosceles triangular enclosure was previously reported by Holtzmann et al. [7] in the case with constant cooling at the inclined surfaces and simultaneous heating at the base. The formation of the asymmetric flow is due to a pitchfork bifurcation as discussed by Ridouanea and Campo [6]

At $t=2.8125 P$, Figure 2(e), the large cell occupies the central region of the cavity. By this time an additional intermittent cell has formed on the top region of the enclosure. The corresponding horizontal plume causes the fluctuation of the Nusselt number apparent in Figure 5.

In Figure 2(f) the large cell has crossed the centreline of the cavity and the smaller cell, closer to this large cell, fills up this gap and grows itself. The flow is then gradually returning to symmetry. The top cell becomes larger and diminishes quickly before the new cycle starts. There are only two cells remaining at the time $t=3.00 \mathrm{P}$, which is the same as that at $t=2.00 \mathrm{P}$ (hence the figure is excluded).

\subsection{Effects of aspect ratio on the flow response}

The flow responses to the periodic thermal forcing for the other two aspect ratios are shown in Figure 3 and 4, which are compared with the flow response for $A=1.0$ shown in Figure 2. We find that the aspect ratio of the enclosure has a great influence on the flow response as well as the heat transfer. The residual effect of the previous cycle on the current cycle is similar for all aspect ratios (Figures 2(a), 3(a) and 4(a)) and the flow and temperature structures during the heating process is qualitatively the same for $A=0.5$ and $A=0.2$ as those for $A=1.0$. However, during the cooling phase there are significant changes of flow and heat transfer among these aspect ratios. The flow remains 
(a) $\mathrm{t}=2.00 \mathrm{P}$
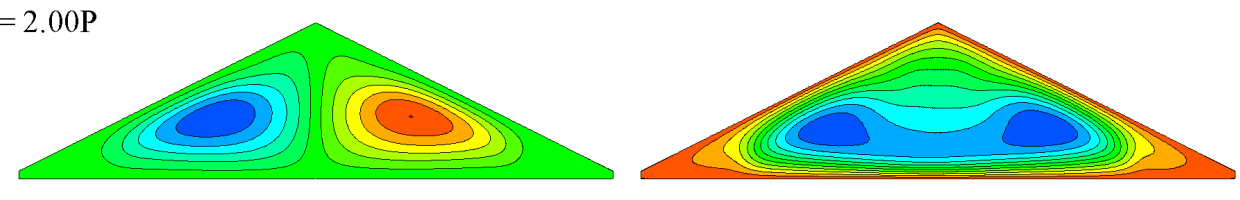

(b) $\mathrm{t}=2.75 \mathrm{P}$
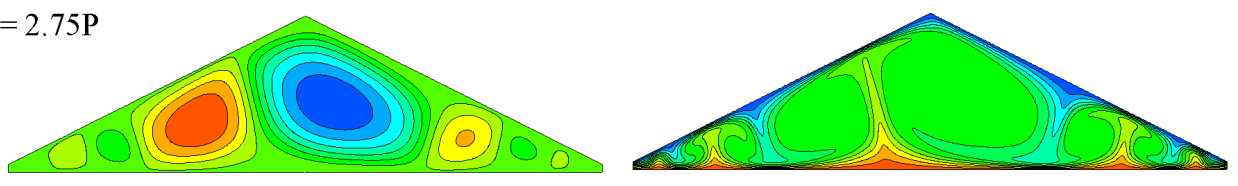

(c) $\mathrm{t}=2.875 \mathrm{P}$
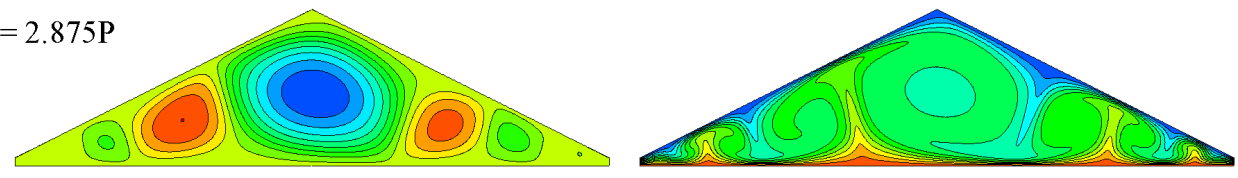

(d) $\mathrm{t}=2.975 \mathrm{P}$
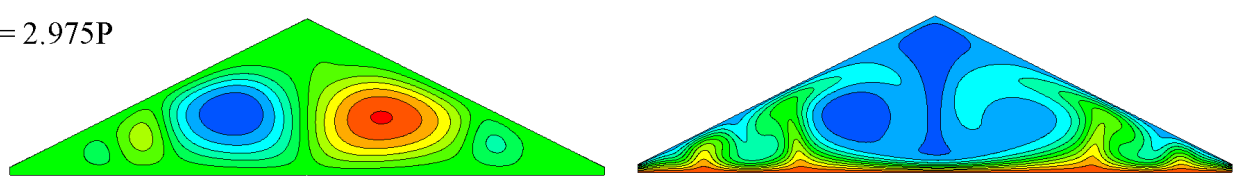

Figure 3: Stream function (left) and isotherms (right) of the third cycle at different times for $A=0.5$ and $\mathrm{Gr}=1.33 \times 10^{6}$. 
(a) $\mathrm{t}=2.00 \mathrm{P}$
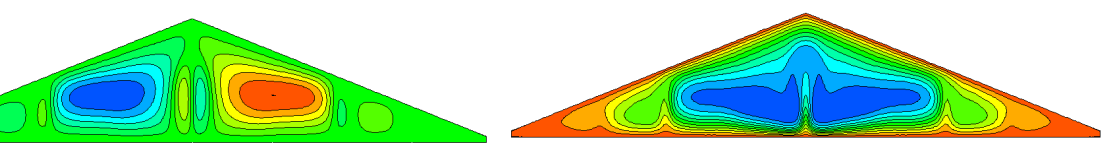

(b) $\mathrm{t}=2.75 \mathrm{P}$
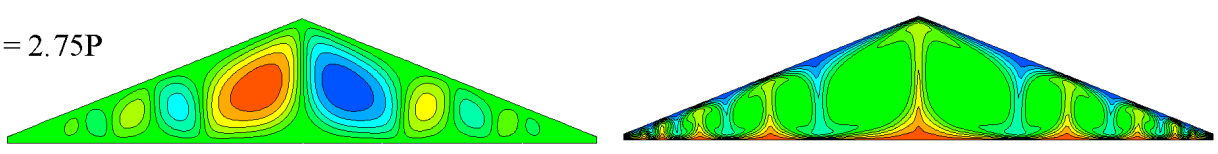

Figure 4: Stream function (left) and isotherms (right) of the third cycle at different times for $A=0.2$ and $\mathrm{Gr}=1.33 \times 10^{6}$.

symmetric about the geometrical centreline throughout the cycle for aspect ratio $A=0.2$, whereas it is asymmetric during the cooling phase for the other two aspect ratios. We anticipate that the asymmetric solution is one of two possible mirror images of the solutions. Another noticeable variation with different aspect ratios is the formation of a circulation cell near the top of the enclosure. For $A=1.0$ that there is an extra vortex, Figure 2(e) and (f), on the top of the cavity, which is completely absent for $A=0.5$, see Figure 3(c), and $A=0.2$.

\subsection{Heat transfer}

The surface Nusselt number, which is a physical quantity measuring heat transfer, is

$$
\mathrm{Nu}=\frac{h_{\mathrm{eff}} H}{\kappa},
$$

where the heat transfer coefficient

$$
h_{\mathrm{eff}}=\frac{q}{T_{A}} .
$$


Here $q$ is the convective heat flux. Since the bottom surface temperature is fixed at $295 \mathrm{~K}$ and the top surface temperature varies between $290 \mathrm{~K}$ and $300 \mathrm{~K}$ in the present simulations, a zero temperature difference between the surfaces occurs twice in a cycle. Therefore, the amplitude of the temperature fluctuation $T_{A}$ is chosen for calculating the heat transfer coefficient instead of a changing temperature difference, which would give an undefined value of the heat transfer coefficient at particular stages.

Figure 5 shows the calculated average Nusselt number on the inclined surfaces of the cavity. The time histories of the calculated Nusselt number exhibit certain significant features. Firstly, they show a periodic behaviour in response to the periodic thermal forcing. Secondly, within each cycle of the flow response, there is a time period with weak heat transfer and a period with intensive heat transfer. The weak heat transfer corresponds to the day-time condition when the heat transfer is dominated by conduction and the strong heat transfer corresponds to the night-time condition when convection dominates the flows and the instabilities occur in the form of rising and sinking plumes. Finally, during the day the heat transfer rate is almost the same for the three aspect ratios. However, at night-time the heat transfer rate for $A=1.0$ is much smaller than that for the other two aspect ratios. This is because the number of the convective cells at $A=1.0$ is smaller than those for the other two aspect aspect ratios, indicating weaker convection at the highest aspect ratio. Also observe that there is a fluctuation of the Nusselt number for a certain period of time. This fluctuation is absent for the other two aspect ratios. The highest average Nusselt numbers for $A=1.0$, 0.5 and 0.2 are $8.06,10.00$ and 9.84 respectively.

\section{Conclusion}

Natural convection in an isosceles triangle subject to periodic thermal forcing has been described in this study. Three aspect ratios 1.0, 0.5 and 0.2 with a 


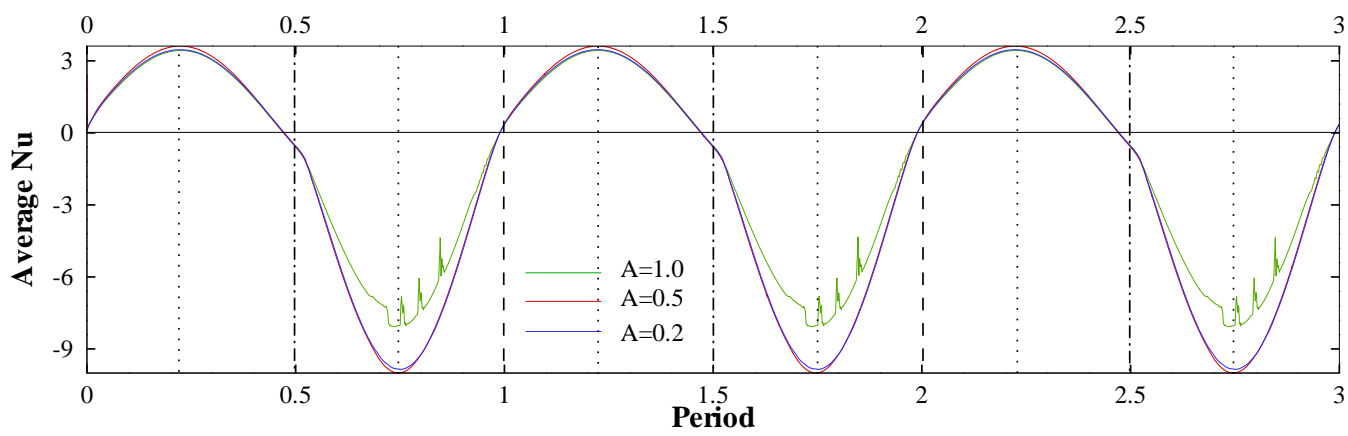

Figure 5: Average Nusselt number on the top surface of the cavity for three full periods where $\mathrm{Gr}=1.33 \times 10^{6}$ and different $A$.

fixed Grashof number $1.33 \times 10^{6}$ and a fixed Prandtl number 0.71 have been considered here. We have found that the flow response to the temperature variation on the external surface is fast, and thus the start-up effect is almost negligible. The occurrence of sinking cold air plumes and rising hot air plumes in the isotherm contours and the formation of cellular flow patterns in the stream function contours confirm the presence of the Benard-type instability. We have also observed that the flow undergoes a transition between symmetry and asymmetry about the geometric symmetry plane over a diurnal cycle for the aspect ratios of $A=1.0$ and 0.5. However, for $A=0.2$, the flow remains symmetric throughout the cycle. Furthermore, the daytime responding flow is weak, whereas the night-time responding flow, which is dominated by convection, is much stronger.

Acknowledgements This research is supported by the Australian Research Council. 


\section{References}

[1] R. D. Flack, Velocity measurements in two natural convection air flows using a laser velocimeter, ASME J. Heat Transfer, 101, 1979, 256-260. C678

[2] R. D. Flack, The experimental measurement of natural convection heat transfer in triangular enclosures heated or cooled from below, ASME J. Heat Transfer, 102, 1980, 770-772. C678, C681

[3] V. A. Akinsete and T. A. Coleman, Heat transfer by steady laminar free convection in triangular enclosures, Int. Journal of Heat and Mass Transfer, 25, 1982, 991-998. doi:10.1016/0017-9310(82)90074-6 C678

[4] D. Poulikakos and A. Bejan, The fluid dynamics of an attic space, J. Fluid Mech., 131, 1983, 251-269. doi:10.1017/S0022112083001317 C678, C681

[5] E. M. Del Campo, M. Sen and E. Ramos, Analysis of laminar natural convection in a triangular enclosure, Numer. Heat Transfer, 13, 1988, 353-372. doi:10.1080/10407788808913618 C679

[6] E. H. Ridouanea and A. Campo, Formation of a pitchfork bifurcation in the thermal convection flow inside an isosceles triangular cavity, Physics of Fluids, 18, 2006, 074102. doi:10.1063/1.2220051 C685

[7] G. A. Holtzmann, R. W. Hill, and K. S. Ball, Laminar natural convection in isosceles triangular enclosures heated from below and symmetrically cooled from above, ASME J. Heat Transfer, 122, 2000, 485-491. doi:10.1115/1.1288707 C679, C685 


\section{Author addresses}

1. S. C. Saha, School of Engineering, James Cook University, Townsville, Queensland 4811 Australia. mailto:Suvash.Saha@jcu.edu.au

2. C. Lei, School of Engineering, James Cook University, Townsville, Queensland 4811 Australia.

3. J. C. Patterson, School of Engineering, James Cook University, Townsville, Queensland 4811 Australia. 